\title{
The digital transformation of physician-patient consultations: Identifying problems and approaches to improve adherence
}

\author{
Andri Färber \\ ZHAW School of \\ Management and Law \\ andri.faerber@zhaw.ch \\ Philipp Stalder \\ ZHAW School of \\ Management and Law \\ philipp.stalder@zhaw.ch
}

\author{
Nicole Zigan \\ ZHAW School of \\ Health Professions \\ nicole.zigan@zhaw.ch \\ Andrea Koppitz \\ ZHAW School of \\ Health Professions \\ andrea.koppitz@zhaw.ch
}

\author{
Mateusz Dolata \\ University of Zurich \\ dolata@ifi.uzh.ch
}

Gerhard Schwabe

University of Zurich

schwabe@ifi.uzh.ch

\begin{abstract}
There is evidence for a correlation between effective physician-patient communication in consultations and improved adherence to treatment. Lack of time, limited communication training, growing administrative duties, and low recall of physicians, information and recommendations by patients are antagonists to effective physician-patient communication. In interviews with physicians, therapists, and patients, we first identify problems of current consultation practices and condense them in a problem scenario. We then use interview results to explore potential solutions, applying modern information technology such as digital medical assistants. Lastly, those potential solutions are condensed in an activity scenario that can be used for further design science research activities.
\end{abstract}

\section{Introduction}

Communication is a central component of a physician-patient consultation [24]. There is evidence for a correlation between effective physician-patient communication and improved adherence to treatment [24]. Thus, effective consultation most likely leads to better adherence to treatment.

In 2012, 78.4\%, or 5.4 million of the 6.8 million, of Switzerland's population visited their physician at least once [5]. This amounted to 13.1 million consultations with physicians and 6.6 million with specialists [5]. Due to the large number of physicianpatient consultations, an improvement in the physician-patient communication will have a significant influence. However, there are antagonists to effective physician-patient communication, such as lack of time [10,15], limited communication training [16], growing administrative duties [15], and low recall by patients of physicians' information and recommendations [20].

The aim of this study is to explore the short- and long-term potential for improving the physicianpatient consultation and thus adherence to treatment with the help of information technology.

The physician-patient consultation as well as its preparation and follow-up are examined. We intend to identify starting points for the design of technological solutions that can be investigated and validated in further research with the help of Design Science Research (DSR) [17, 26]. We use scenarios to make problems and their potential solutions visible and tangible [30].

\section{Problem scenario}

This section presents an introductory "problem scenario" about current physician-patient consultations [30]. It is based on the literature analysis and interview results as described further below. Identified problems are italicized.

Giovanni is 60 years old and immigrated from Italy to Switzerland 23 years ago. Since the death of his wife three years ago, he has been living alone in his small but attractive apartment in the countryside near Zurich. For three months, Giovanni has been suffering from recurring headaches. Three weeks ago, his general practitioner referred him to Dr. Smith, a neurologist. Both Giovanni and Dr. Smith remember this first consultation well. Giovanni came to the appointment rather nervously and wanted Dr. Smith to fully comprehend his stressful situation. He began to describe his illnesses, symptoms, and living conditions in detail. It took Dr. Smith much longer than planned to understand and 
identify Giovanni's main health concern. In the course of the conversation, Giovanni managed to focus and build trust with Dr. Smith. After this initial assessment, the physician sent Giovanni home with a painkiller, a diary to record the frequency and severity of his headaches, and recommendations on how to adjust his behavior. As the pain did not subside, Giovanni made a second appointment with Dr. Smith.

During the days before his second consultation, Giovanni googled intensively symptoms and diagnoses on what he could ask Dr. Smith. In order to remember everything, he wrote the questions neatly on a piece of paper. Shortly before leaving, he remembered that he had not filled out the diary given to him by Dr. Smith during their first meeting. He tried as best as he could to remember the occurrences of his headaches since the last consultation, and made records in the diary accordingly. He did not really understand why he had to do it. He was hardly finished when Maria, his daughter, rang the doorbell. She had offered to drive him to his appointment with Dr. Smith.

After a warm welcome, Dr. Smith skimmed over Giovanni's medical file while asking Giovanni to tell her how he was feeling today. Giovanni revealed to her his frustration about his ongoing headaches and expressed his worst fears based on his Internet research. Dr. Smith spent the first 10 minutes reassuring Giovanni and explaining to him that the probability of suffering from a malignant brain tumor was negligible. Relieved, Giovanni then showed Dr. Smith the completed diary. Upon persistent inquiries from Dr. Smith, he admitted that he had not completed the diary until just before the appointment. Dr. Smith showed Giovanni the results of the last examinations, turning the screen in front of her so that Giovanni could also see it. The confusing value tables and complicated graphics did not tell Giovanni very much, however, and although Dr. Smith tried to explain the facts to Giovanni in simple words, he still did not understand. Dr. Smith would have liked to show Giovanni the image of a brain to show him where she suspected the cause of the headache to be. Unfortunately, she could not find it in her bookshelf or on her computer within a reasonable period of time and therefore made do without $i t$. Since Dr. Smith had come to know Giovanni as a very interested and self-determined patient, she discussed the next steps with him in detail and adapted it according to Giovanni's wishes. A small detail in Giovanni's remarks should have prompted Dr. Smith to listen attentively; however, distracted by a short phone call from her assistant, it was lost. As a direct result, the correct diagnosis could not yet be made. In preparation for the next consultation, Dr. Smith asked Giovanni to make entries in his diary more regularly. She also provided a printout of the tables and graphs shown on the screen and oral instructions for relaxation exercises to be performed three times a day.

After Dr. Smith said goodbye to Giovanni, she used her handwritten notes from the conversation to dictate the most important details and the next steps for treatment.

On their way home, Giovanni told Maria about his conversation with Dr. Smith. He realized that he had forgotten to refer to his note and to ask the questions he had written down beforehand. Maria asked what advice Dr. Smith had given for dealing with the headaches.
Unfortunately, Giovanni remembered very little of what Dr. Smith had said.

\section{Related Work}

\subsection{Physician-patient communication}

For centuries, responsibility for the problems and treatment of patients has rested exclusively with physicians [3]. In this traditional setting, compliance in or adherence to treatment is generally poor [36]. Two new concepts or paradigms attempt to change this: patient empowerment and shared decision making. Therefore, if we intend to support physicianpatient communication with information technology, then such solutions must support patient empowerment and shared decision making. Before we introduce these two concepts, a brief overview of the development of adherence to treatment is given.

The term "compliance" emerged in the 1970s from a paternalistic understanding of the physician-patient relationship [22]. Compliance in this paradigm means that patients follow their physicians' instructions. One of the underlying assumptions is that the patient is the cause of the problem in case of non-compliance [3]. While this paradigm may work in acute cases, it is not suitable for the treatment of chronic diseases and longterm care. With the term "adherence," a new paradigm was introduced. It defines patients "as independent, intelligent, and autonomous people who take more active and voluntary roles in defining and pursuing goals for their medical treatment" [23]. As long as adherence is seen merely as a characteristic of individual patients, however, it does not make a significant difference to compliance. The difference is made by physicians communicating more openly and working more collaboratively with their patients [23].

Working collaboratively with the patient is the core of patient empowerment [6]. It is based on (1) the co-creation of knowledge during an effective dialog between the patient and the health care provider, (2) a patient-centered approach, (3) a sufficient level of health literacy, and (4) active participation [6].

In real shared decision-making physicians and patients decide jointly on the best course of treatment. Physicians provide their expertise while patients express their preferences in a two-way exchange [7].

In summary, shared decision making and patient empowerment are not something that is done to the patient. It is not "convincing, persuading, "empowering,' or changing patients (or getting them to change)" [4]. It requires that both sides become involved and come to a shared understanding and decision [7]. 


\subsection{Impact of the physician-patient communication on adherence to treatment}

Improving physician-patient communication cannot be an end in itself. The very expensive health systems simply lack the money for this. It must therefore be proven that enhancing physician-patient communication is effective, e.g., by improving adherence to treatment.

$30-50 \%$ of all patients adhere badly to treatment [36]. There seem to be different levels of adherence in different forms of therapy. In diabetes care, $91 \%$ of the patients took their medication, 52\% followed a diet, and only $19 \%$ participated in an exercise program [20]. Physician-patient communication and physician training in communication skills are shown to have a significant impact on patients' adherence to treatment [37]. A further improvement is likely to result from patients' abilities to recall physicians' information and recommendations. Among patients with diabetes, the recall of recommendations ranges from $96 \%$ (recall of prescribed medication) to $50 \%$ (recall of instructions on various aspects of diabetes self-care) [20].

\subsection{Existing Solutions}

Research in the field of medical informatics studies the use of existing technologies in physician-patient communication. Among others, it focuses on how physicians employ shared displays of various forms (wall screens, tablets, desktop monitors) to show electronic medical records to patients or to gather the necessary information in a collaborative way $[2,11$, $21,25,28]$. While the studies do not provide a conclusive answer as to whether computers improve the physician-patient interaction or not [2, 11, 28], they make clear that the use of technology is growing - reading or filling out medical records amounts to $25 \%$ of consultation time and covers over 40 specific activities [21]. Physicians also record their consultations in an audio or video format to make the documentation processes less interruptive for the communication with the patients, although this practice remains controversial [29]. Technologies expected to enter the consultations are double checks / clinical diagnosis decision support systems in which AI supports the physician with the interpretation of proper symptoms and treatment choice, but how they will impact the interaction with the patient remains unclear [33]. Also, the popularity of mHealth, mobile health applications which support self-monitoring and self-management by patients, may contribute to the extended presence of computers in consultations and may support adherence $[1,19]$. Overall, the impact of computers on physician-patient communication in consultations is expected to grow: ICT has the potential to enhance documentation, seamlessly integrate and process patient data (also from mobile applications), suggest better treatments, and help to visualize the content. However, the available systems are mostly isolated, focus on the primary tasks (such as documentation in electronic records), and attach limited importance to their impact on the interaction between physician and patient. This leads to lower credibility [28], faltering conversations [25], or negative effects related to physicians' listening behaviors [11], all of which may reduce patients' adherence to treatment.

Nevertheless, computer use during a collaborative encounter may produce practical gain (better documentation or visualization) while also positively impacting the interpersonal layer. Research on advisory services in financial institutions [12], travel agencies [31], and police forces [9] exemplifies that careful design which acknowledges the highly sensitive nature of collaboration practices helps for the advisor and the advisee to be more satisfied and for the advisee more likely to follow the advice. In particular, employing elements of persuasive design in police forces' burglary prevention services bears the potential to enhance recommendation adherence [9, 13]. Such efforts suggest a direction for further research in the medical domain. However, it remains unclear whether they can be adapted to the higher complexity and variety of topics in physician-patient interaction, as well as the physicians' specific practices.

\section{Methods}

The overall research approach follows the scenario-based design technique [30] and implements it in the context of design science research $[17,26]$. We argue that scenarios, as a form of contextualized narratives, are well suited to capturing problems and solutions in a comprehensive and illustrative manner [30]. A scenario allows for validation of the common understanding of the problems in discussions with experts or users and for the improvement of the solution description; it also forms a boundary object within a research team and beyond it [30]. We use the collaboratively-written and literature-inspired scenarios throughout this study to describe problems of healthcare communication, validate the understanding of those problems with healthcare professionals and patients, develop a vision of a potential solution, and, finally, evaluate this vision. 
Based on the initial literature research as well as workshops with experts in the field of computersupported collaboration, medicine, and healthcare science, a set of problems and solution scenarios emerged. Those scenarios summarized the issues concerning communication between stakeholders in the healthcare domain which were reported in the literature and by the experts from the perspective of a patient. The scenarios formed the basis for further specification of the most impactful issues and most promising improvement potentials.

To further explore and understand the challenges from the practitioners' perspective, we engaged in interviews with Swiss physicians, therapists and patients ${ }^{1}$. In particular, we focused (as in the literature research) on the following topics: (1) physicianpatient communication and (2) the impact of the physician-patient communication on adherence to treatment. We conducted semi-structured interviews [32] with eight physicians from various professions (general practitioners, neurologists, dermatologists, hematologists, diabetologists) with varying levels of experience (between 40 and 76 years of age) and of different genders (three male and five female), and with seven patients (aged between 48 and 77, two male and five female) with different health conditions. We also conducted interviews with two physiotherapists and one dietician to learn their opinions about potential improvements in professsional-patient communication. Five patients were recruited through a flyer. Two patients were found through convenience sampling. Two general practitioners, the diabetologist, and the dietician were nominated by their patients. All other health care professionals were recruited through convenience sampling. Two neurologists and one physiotherapist work at the same hospital. The diabetologist and the dietician work door to door at the same clinic. All other health care professionals work independently of each other at different locations. During the interviews, the study participants were first asked a set of open questions based on the literature about physician-patient communication, then asked a set of questions approaching critical incidents from the past [14, 18], and lastly, were confronted with the prepared scenarios. This allowed for the collection of independent opinions and the evaluation of the scenarios.

The collected data was qualitatively coded using Atlas.ti in a bottom-up manner following a contentanalytical and practice-oriented approach [8]. The coding procedure resulted in the identification of 10 distinct/overlapping areas describing an urgent need

\footnotetext{
${ }^{1}$ Switzerland has one of the most effective but also most expensive health systems in the world [27]. We therefore
}

for improvement. The subsequent section describes those areas in more detail. The final scenarios were adapted to those insights and describe the problems validated by the stakeholders as well as the solutions considered most adequate by them.

\section{Results}

The interviews showed that patients and health care professionals such as physicians and therapists have experiences and expectations in relation to the professional-patient consultation; they also reveal the ICT support during the face-to-face consultation as well as the preparation and follow-up of consultation.

\subsection{Supporting the consultation}

The following relevant topics emerged in relation to the consultation and ICT-support: relationship, facilitation, documentation, double checks, patient education, and recall of information and recommendations.

Relationship: For both the patients and the physicians, relationship building is elementary during the consultation. It is important to the patients that they are treated seriously and respectfully, and that they feel well-understood. It is not enough for them to merely be treated well medically. The physicians place importance on building a team with their patients. They assess patients not only medically but also on a personal level to find an appropriate degree of conversation for shared understanding so that patients can participate. The physicians in particular reported that long-lasting relationships with their patients are an important element of their job satisfaction. One physician described the relationship as "dancing with the patient instead of fighting" (phy2).

Facilitation: The physicians typically follow a structured procedure to facilitate their consultations. For example, they use paper-based questionnaires for the initial assessment, which patients complete prior to the consultation. Others reported using standardized questions during their consultation. The patients appreciate the opportunity to ask questions and explain how they are doing. "But to merge all problems to one point is relatively challenging." (phy. 4). On their desks, the physicians tend to have computers whose screens are visually inaccessible to patients, and the physicians decide whether to turn their screens in the direction of the patients. The physicians judge the types of adherence of the patients differently: higher

believe that this study is relevant beyond Switzerland's borders. 
in taking the medication and lower in performing exercises or making lifestyle changes. Patients confirmed this as well.

Documentation: The documentation of the consultation is most often very time-consuming for the physicians. The use of the computer for administrative purposes is common but varies greatly during the consultation. The patients feel disturbed during consultations only if physicians are unable to use the computer properly. Some physicians prefer handwritten notes during their consultations because of the complicated, pre-structured clinic information systems or electronic health records. Many dictate their notes with automatic transcription afterwards, delegate it to their assistants, or type the notes directly into the computer. The patients generally document little during the consultations, except with regard to unknown drug names, complex drug dosages or relevant information sources recommended by their physicians. The physicians and most patients would welcome an automatic recording, a transcription, and an analysis of their communication. "That would be great, that would be perfect, yeah (laughs). I'd install it right away." (phy. 7)

Double checks: The physicians already use electronically available expertise during consultations. Looking up side effects of drugs and drug interactions is part of their everyday work. In addition, they search for information or confirm their decisions using the Internet pragmatically, for example to look up necessary vaccinations for travel, differential diagnoses, memory tests, and similar topics. A few patients also accepted invitations from pharmacies or their health insurance companies to check their drugs.

Most of the interviewed physicians would appreciate the help of a digital medical assistant, which would go much further and, based on all available medical literature, could draw physicians' attention to differential diagnoses and forgotten questions or tests at the right moment. It is important for most that the digital medical assistant does not enter into a direct dialogue with the patient, however, for two main reasons: fear of loss of control or credibility, and keeping control of the information that the patient receives. "I think the most difficult thing, I can imagine, for a patient are the unfiltered diagnoses, (...) they need a weighting by physicians. But then I think it's a good thing" (phy. 1). Most of the patients appreciate that the digital medical assistant ultimately supports the physicians' decisions. They trust the physicians to handle their data securely.

Patient education: The physicians take on the challenges of informing patients about their complex situations and educating them as best as possible under the time-restricted circumstances. Strongly dependent on the respective discipline, the physicians already use various aids to explain diagnoses and possible therapies to their patients. The aids used in consultations include pictures, models, laboratory values, tables, graphics, guidelines, and, above all, drawings. The more specialized a discipline and the smaller the number of possible diagnoses and therapies, the more aids are used. In the limited consultation time available to them, they often do not find the appropriate aid within a useful time limit.

Based on the patients' experience, it is uncommon to have visual aids during each conversation. The patients are impressed and feel appreciated when physicians take the time to explain their conditions using pictures or graphs, whether paper-based or on the screen. "Now I can imagine it -from the pictures, he showed me. And tell myself, that if I resign now and go again the more comfortable way, it will happen again. (...). And then that (the pain) will come again." (pat. 7)

Most patients would prefer to have more shared views, such as laboratory value progression over time, to better understand the association to their medication regimes or lifestyle changes.

Recall of information and recommendations: Despite oral summaries of relevant information given by the physicians, sometimes added with further information brochures or websites, the physicians often have to repeat the same information in consecutive consultations. The patients stated that they can normally remember the information, with help of their notes, physicians' notes, the prescription, or the received material to adhere as best as possible to the therapy regimes and life style changes recommended. The patients reported that they keep the documents with recommendations related to therapy goals as a mental reminder. "I put them in this box and if it didn't go well, then: Oh, what did she write down? Then I'll go and see..." (pat. 1)

Most of the interviewed patients as well as the physicians endorsed the suggestion that a digital medical assistant generates a "physician-patient consultation to take home". It would be supportive for the patients to receive a short summary of the consultation, added with physicians' individualized adaptions and recommendations. "Because (the physician) tells me that, I can't remember when I walk out the door. That would be helpful (...) you could look it up again" (pat. 1)

\subsection{Supporting the preparation and follow-up of the consultation}

The following relevant topics emerged in relation to the ICT-support in preparation and follow-up of 
consultations: patient education, instruction, monitoring, and interaction.

Patient education: Most of the interviewed physicians complained about patients being misinformed through the vast amount of information freely available on the Internet before and/or after consultations. The physicians spend a considerable amount of time calming patients down and providing them with the right information. Therefore, some physicians advise their patients against searching the Internet and provide them with links to websites with correct information about the right diagnoses and therapies. The patients appreciate the opportunity to take home physicians' drawings from consultations; however, most of the interviewed patients reported a lack of received information material, especially at the beginning of the chronic trajectory. They conducted their own searches through various sources, including journals, support groups, and often the Internet. As a result of negative experiences, the patients wish to have more information about which sources can be trusted. "Of course, I searched on the Internet, what can you do (...). There are many forums: Whereby, I someday stopped, because it is sometimes depressing." (pat. 5).

Instruction: Both, the physicians and the therapists use a broad range of instructional material, as well as written therapy protocols and goals. In a creative manner, one therapist stated that he invites patients to videotape their exercise lectures using their own smartphones. "It's much more effective before I start drawing or downloading anything. I record this for the patient and then he has his exercise program on his smartphone" (ther. 2). The patients use the received material or the internet to find instructions. One patient found a suitable conversion table for insulin dosages on the internet. Another patient searched for relaxation exercises, but struggled to perform the exercises correctly.

Monitoring: The physicians and the therapists use diverse questionnaires, diaries and devices for monitoring purposes. The patients try to find the best way to gain control over their chronic diseases. One patient did not feel rewarded enough for her monitoring of her activities, as errors were frequently highlighted in the consultation, and she did not feel adequately supported. One patient started to regularly document on paper the medication intake and level of pain on his own accord. Retrospectively, the patient regrets not having done it systematically and not having kept it. The patient stated that a diary would have helped in his situation, as well as a graphical view, in which more than one parameter is shown over a long period of time. Other patients reported using the computer or disease-specific applications to document parameters and laboratory values, sometimes without involving their physicians. One patient would like the results on his smartphone explained by the app. "No, I think it's more of a self-control, (...) to see for myself: Oh, now the (parameter) is - uh. And that annoys me when the curve - but this is purely personal. (...) It gives me no perspective, (...) no comment what, what I - how am I. How good or qualitatively good are these values?" (pat. 3). Most patients are open to trying new technologies and would invest in a new smartphone, for example. Several patients wish to have lab results to take home.

Interaction: Some patients wrote down questions to prepare themselves for their consultations. Between the consultations, they sometimes used phone calls for short forgotten questions or in cases where a promised callback from the physician did not take place. Some patients contacted their physicians by email or post to describe their progress or to report how they had adapted the therapy on their own. The patients can imagine using a smartphone or tablet app to document questions for the next consultation or to fill out a necessary questionnaire. "Well, yes - it might be a good idea just send an e-mail to the physician to be prepared. That would be - so he doesn't have to ask for a long time (...)" (pat. 2).

\section{Discussion}

The design/activity scenario below tells the story of a physician-patient consultation after the introduction of the new technology [30]. Identified solutions are italicized. Selected solutions and findings are discussed in more detail following the design/activity scenario.

\subsection{Design/activity scenario}

As an energetic and enterprising person with a high need for autonomy, Giovanni finally wants to get his headaches under control. Therefore, since his last appointment, he painstakingly follows the recommendations for behavioral change in adherence to Dr. Smith's instructions. The tutorial on his My Doctor app is a great help. In the first several days, Giovanni fails to do certain exercises perfectly. The feedback from the app based on the video recording of his exercises enables him to correct this quickly. Three times a day, the app draws Giovanni's attention to the fact that he should update his pain diary. With two or three clicks, Giovanni can pinpoint his pain and indicate its intensity on a scale from 0 to 10 . Giovanni then declares his respective emotional state simply by selecting one of five emoticons. In addition to the instructions and feedback on his exercises, Giovanni finds background information about his probable diagnosis on his app. Questions and concerns that he may 
have can be entered directly into the app at the appropriate points, either via the on-screen keyboard or by voice input.

On the day of the doctor's appointment, Giovanni is picked up at home by Maria, his daughter, and driven to Dr. Smith. During their conversation about the upcoming consultation, Maria raises additional questions which Giovanni also dictates into his My Doctor app.

In the doctor's office, Dr. Smith warmly welcomes Giovanni and asks him to sit down. Dr. Smith and Giovanni sit at a 60-degree angle to each other. In this way, they can see each other and at the same time view the large screen on the wall. Dr. Smith asks Giovanni whether he agrees for their conversation to be recorded, transcribed, and analyzed electronically. Giovanni has confidence in technology and consents. After engaging in small talk, Dr. Smith shows Giovanni the evaluation of his diary and home exercises as well as the examination results from his last consultation on the wall screen. Based on certain assumptions made about the exact diagnosis, she asks about individual diary entries in detail. Uncertain about the diagnosis, Dr. Smith notices the discreet reference of the digital medical assistant on her screen. It recommends that she ask Giovanni two or three very specific questions. Only now does Giovanni remember the minor fall in the bathroom three months ago. Suddenly, everything becomes clear. The headaches are almost certainly the result of a craniocerebral trauma caused by this fall. Dr. Smith explains to Giovanni what happens to the brain in such a case. The digital medical assistant offers her two matching images and a video sequence from its collection, which Dr. Smith then presents to Giovanni on the wall monitor. Dr. Smith explains to Giovanni what she believes should be changed in Giovanni's therapy based on this diagnosis. Together they define the therapy goals as well as the necessary exercises and diary entries. Based on the course of the discussion so far, the digital medical assistant generates for Dr. Smith a suggestion for the "physicianpatient consultation to take home". This consists of multimedia-based background information on the diagnosis made and the corresponding therapy options, the therapy goals and the instructions for the exercises and diary entries. All this can be called up in words understandable for laymen.

On the way home, Giovanni tells Maria about his conversation with Dr. Smith. At home, with Giovanni's permission, Maria watches the "physician-patient consultation to take home". By doing so, she learns several behavioral tips which Giovanni could not remember during their drive back.

In the coming days and weeks, Giovanni does the agreed exercises conscientiously with the support of the My Doctor app, which leads to a rapid improvement of his medical condition. Giovanni can send short questions about his therapy via the app to Dr. Smith either through a typed message or by video message. He typically receives a competent answer from Dr. Smith within 24 hours.

\subsection{Supporting the consultation}

We discuss below how a physician-patient consultation can be supported by ICT in the previously identified areas: relationship, facilitation, documentation, double checks, patient education, and recall of information and recommendations.

Relationship: Literature [6, 7] and interviewed physicians, therapists, and patients agree that relationship building is essential to enable patient empowerment and shared decision making. Although there are indications that computers have an influence on physician-patient interaction [11, 28], it is still unclear if and how they affect the physician-patient relationship [2]. For the time being, it can be assumed that ICT can at least free time that can be invested in relationship building. Future research may focus on how information technology can directly support relationship building between physician and patient.

Facilitation: The facilitation of the consultation has become more demanding for the physician with the concepts of patient empowerment [6] and shared decision making [7]. There are many ways in which computers can support facilitation, communication, and patient education [2, 9, 11-13]. The interviewed physicians, therapists, and patients are open to computer support as long as the computer adapts to the nature of human interaction and not vice versa. Physicians, therapists, and patients reported that it is not only important what is said during the consultation, but how it is "sold" to the patient as well. For example, the computer could support "selling" recommendations for lifestyle changes with attractive pictures and professional-looking charts. Future research could investigate the effect of such "marketing tools" on adherence to treatment.

Documentation: The proposed automatic recording, transcription and analysis of the consultation promises significant time savings and a comprehensive recording of what was discussed. The time gained could be used for patient treatment $[10,15,16]$. It also prevents the loss of important details and would also be advantageous in the case of liability claims. It is imperative that an intelligent, automated analysis and summary of the consultation is provided, since very few physicians would find time to read extensive interview transcripts.

Double checks: A digital medical assistant as described in the solution scenario would allow double checks without consuming additional time $[10,15$, 16]. Double checks would presumably be made more frequently, which would subsequently lead to better health outcomes [33]. The interviewed dietician was the only one open to the idea of the digital medical expert participating directly in the conversation - with a remarkable thought. She would see it as a sparring partner, which would even allow her to direct a patient's bad feelings, for example due to food restrictions, away from her and onto the computer. 
Further research could investigate the impact of an active digital medical expert as a third participant in the consultation.

Patient education: If a digital medical assistant listened to the conversation and offered the physician an adequate aid at the right moment, then there would be technological support for all the patient empowerment and shared decision making based on $[4,6,7]$ (the co-creation of knowledge during an effective dialog between the patient and the health care provider, a patient-centered approach, a sufficient level of health literacy, and active participation [6]). Here, too, time is a critical success factor. For the use of patient education aids, it is crucial to have the right aid available at the right time; otherwise, it is not used.

Recall of information and recommendations: The literature [20] and the physicians, therapists, and some patients interviewed speak of a low percentage of recall of physicians' and therapists' information and recommendations. This has a direct negative effect on adherence to treatment [20]. Physicians report that they have to continuously start over again from one consultation to the next. The "physician-patient consultation to take home" offers a means to change that. Based on all available medical literature and on what has been discussed between physician and patient, a digital medical assistant may suggest to the physician what information and recommendation the "physician-patient consultation to take home" should comprise. The physician then decides what is actually given to the patient. This addresses the physicians' concerns mentioned in [29].

\subsection{Supporting the preparation and follow-up of the consultation / closing the loop}

Physicians and patients complain about limited consultation time, particularly when it impedes patient education. This may be one reason for patients' very low reported recall of physicians' information and recommendations, which requires time-consuming repetition of details at the next consultation. Many physicians try to prevent this by supporting the aftercare of patients with drawings, handwritten notes, or brochures. This has led to the introduction of a more sophisticated solution in the design/activity scenario the "My Doctor app". It was well received by most people interviewed. The app's main task is to close the loop between the physician-patient consultation, its preparation, and its follow-up for all those involved (see Figure 1). This app should support the following elements of patient empowerment: a patient-centered approach, a sufficient level of health literacy, and active participation [6]. The acceptance of such an app will be increased if it is evidence-based and developed in cooperation with health care professionals [1]. While there are already various patient apps on the market, most are isolated from physicians' information systems. The interviewed physicians stressed the importance of such an app being seamlessly integrated into their IT infrastructure to avoid time-consuming manual data transfer and data inconsistencies.

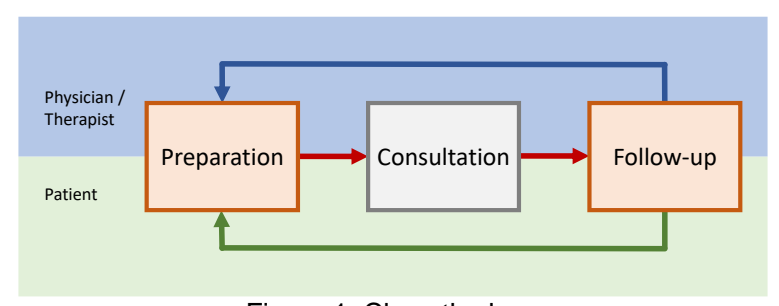

Figure 1: Close the loop

We identified four main functions for the My Doctor app from our analysis of the literature and from the coding of our interviews. The four functions are: patient education, instruction, monitoring, and interaction.

Patient education: Health literacy is one of the prerequisites for patient empowerment [6]. Physicians explaining their diagnosis and therapy to their patients in a comprehensible form can improve health literacy. However, this is challenging within the limited consultation time. Therefore, the first and most important main function of the app is to educate the patient, thus increasing his or her health literacy. It should simultaneously prevent patients from being misinformed, particularly through the Internet.

Instruction: The second main function of the My Doctor app is instruction. Patients forget much of what was discussed during consultations [20]. The solution is the "physician-patient consultation to take home" mentioned in the previous section. Its purpose is to increase patients' recall of information and recommendations. It also allows medical terminology used by physicians to be translated into laymen's terms. Instructions can be given on the app in the form of text, audio, pictures, drawings, video, and more. Instructions mainly cover medication, exercises, diets, and lifestyle changes.

Monitoring: The third main function of the My Doctor app is monitoring. Once the patient has agreed on and understood all instructions, it is important to adhere to them [36]. Monitoring has two objectives.

Firstly, it should support the patient in following the instructions. There may be reminders for taking medication at the right time that the patient needs to confirm; diaries in which the patient enters how severe his or her pain is under what circumstances and/or at 
what times; nudging mechanisms that support the patient in making lifestyle changes; or a video monitoring function to help the patient perform exercises correctly. There could be many other monitoring functions, including vital parameters. With all, it is essential that the patient knows what is being monitored and consents to it.

Secondly, the results of patient monitoring should be fed back to the physician and the patient. This is an important element to close the loop from the followup of the last physician-patient consultation to the preparation of the next. The monitoring results inform about the patient's adherence to treatment, the statistically analyzed content of diary entries, and other monitored measures.

Interaction: The fourth main function of the My Doctor app is interaction. It allows patients to note questions and comments wherever they are. They may even see if their physician is available for a phone call. In return, it may allow physicians to contact their patients in case of a dangerous situation that is detected by monitoring or to support them in their therapy.

\section{Conclusions and Limitations}

Patient education and active participation are key factors in improving adherence to treatment. Both concepts can be supported by human- or patientcentered ICT solutions that close the loop from one consultation to the next and interweave physicians' software with patients' apps. Therefore, further research should focus on extending today's isolated and documentation-centric applications to integrated patient-centered solutions.

The physicians, therapists, and patients interviewed are highly motivated to get the best out of the physician-patient consultation. They have little or no concerns regarding new technologies and data protection - on the contrary. Technology that supports the consultation in a user-friendly and easy-to-use way would be very welcome. There is one hard condition on the part of the physicians and therapists, however: Any form of technological support must not lead to a more time-consuming consultation process.

This research comes with a few limitations: physician-patient communication might not be exactly the same in all countries [34, 35]. Further research could separate generic physician-patient communication elements from country-specific ones. There may also be a selection bias in the patients who were recruited via a flyer; for example, patients with above-average interest and motivation may have responded.

\section{References}

[1] Ahmed, I., N.S. Ahmad, S. Ali, S. Ali, A. George, H. Saleem Danish, E. Uppal, J. Soo, M.H. Mobasheri, D. King, B. Cox, and A. Darzi, "Medication Adherence Apps: Review and Content Analysis", JMIR mHealth and uHealth, 6(3), 2018, e62.

[2] Alkureishi, M.A., W.W. Lee, M. Lyons, V.G. Press, S. Imam, A. Nkansah-Amankra, D. Werner, and V.M. Arora, "Impact of Electronic Medical Record Use on the PatientDoctor Relationship and Communication: A Systematic Review", Journal of general internal medicine, 31(5), 2016, pp. 548-560.

[3] Anderson, R.M. and M.M. Funnell, "Patient empowerment: Reflections on the challenge of fostering the adoption of a new paradigm", Patient Education and Counseling, 57(2), 2005, pp. 153-157.

[4] Anderson, R.M. and M.M. Funnell, "Patient empowerment: Myths and misconceptions", Patient Education and Counseling, 79(3), 2010, pp. 277-282.

[5] https://www.bfs.admin.ch/bfs/de/home/statistiken/gesundhe it/gesundheitswesen/artzpraxen.html, accessed 5-21-2018.

[6] Castro, E.M., T. van Regenmortel, K. Vanhaecht, W. Sermeus, and A. van Hecke, "Patient empowerment, patient participation and patient-centeredness in hospital care: A concept analysis based on a literature review", Patient Education and Counseling, 99(12), 2016, pp. 1923-1939.

[7] Charles, C., A. Gafni, and T. Whelan, "Shared decisionmaking in the medical encounter: What does it mean? (or it takes at least two to tango)", Social Science \& Medicine, 44(5), 1997, pp. 681-692.

[8] Chughtai, H. and M.D. Myers, "Entering the field in qualitative field research: A rite of passage into a complex practice world", Information Systems Journal, 27(6), 2017, pp. 795-817.

[9] Comes, T. and G. Schwabe, "HOW TO DIMINISH ADVICE DISCOUNTING WITH MOBILE MULTIMEDIA INTERVENTIONS", Research Papers, 2016.

[10] Costello, K.L., "Impact of patient-provider communication on online health information behaviors in chronic illness", Proceedings of the Association for Information Science and Technology, 53(1), 2016, pp. 110.

[11] Crampton, N.H., S. Reis, and A. Shachak, "Computers in the clinical encounter: A scoping review and thematic analysis", Journal of the American Medical Informatics Association : JAMIA, 23(3), 2016, pp. 654-665.

[12] Dolata, M. and G. Schwabe, "Tuning in to More Interactivity - Learning from IT Support for Advisory Service Encounters", i-com, 16(1), 2017, p. 202. 
[13] Dolata, M. and G. Schwabe, "Don't Be Afraid! Persuasive Practices in the Wild", J. Computer Supported Cooperative Work (CSCW), 2018.

[14] Flanagan, J.C., "The critical incident technique", Psychological Bulletin, 51(4), 1954, pp. 327-358.

[15] Golder, L., C. Jans, S. Tschöpe, A. Venetz, and N. Herzog, Verändertes Arbeitsumfeld und Einstellung zu neuen Finanzierungsmodellen: Auswirkungen Leistungsorientierung im Gesundheitswesen erkennbar, gfs.bern, 2018.

[16] Hemmer-Schanze, C. and H.S. Füessl, "Wer verstehen will, braucht Zeit", MMW - Fortschritte der Medizin, 148(3), 2006, pp. 25-31.

[17] Hevner, March, Park, and Ram, "Design Science in Information Systems Research", MIS Quarterly, 28(1), 2004 , p. 75.

[18] Keatinge, D., "Versatility and flexibility: Attributes of the Critical Incident Technique in nursing research", Nursing and Health Sciences, 4(1-2), 2002, pp. 33-39.

[19] Kotecha, D., W.W.L. Chua, L. Fabritz, J. Hendriks, B. Casadei, U. Schotten, P. Vardas, H. Heidbuchel, V. Dean, and P. Kirchhof, "European Society of Cardiology smartphone and tablet applications for patients with atrial fibrillation and their health care providers", Europace : European pacing, arrhythmias, and cardiac electrophysiology : journal of the working groups on cardiac pacing, arrhythmias, and cardiac cellular electrophysiology of the European Society of Cardiology, 20(2), 2018, pp. 225-233.

[20] Kravitz, R.L., R.D. Hays, C.D. Sherbourne, M.R. Dimatteo, W.H. Rogers, L. Ordway, and S. Greenfield, "Recall of Recommendations and Adherence to Advice Among Patients With Chronic Medical Conditions", Archives of Internal Medicine, 153(16), 1993, pp. 18691878.

[21] Kumarapeli, P. and S. de Lusignan, "Using the computer in the clinical consultation; setting the stage, reviewing, recording, and taking actions: Multi-channel video study", Journal of the American Medical Informatics Association : JAMIA, 20(e1), 2013, e67-75.

[22] Lerner, B.H., "From careless consumptives to recalcitrant patients: The historical construction of noncompliance", Social Science \& Medicine, 45(9), 1997, pp. 1423-1431.

[23] Lutfey, K.E. and W.J. Wishner, "Beyond "compliance" is "adherence". Improving the prospect of diabetes care", Diabetes Care, 22(4), 1999, pp. 635-639.

[24] Matusitz, J. and J. Spear, "Effective doctor-patient communication: An updated examination", Social work in public health, 29(3), 2014, pp. 252-266.

[25] Pearce, C., M. Arnold, C.B. Phillips, S. Trumble, and K. Dwan, "The many faces of the computer: An analysis of clinical software in the primary care consultation",
International journal of medical informatics, 81(7), 2012, pp. $475-484$.

[26] Peffers, K., T. Tuunanen, M.A. Rothenberger, and S. Chatterjee, "A Design Science Research Methodology for Information Systems Research", Journal of Management Information Systems, 24(3), 2007, pp. 45-77.

[27] Pietro, C. de, P. Camenzind, I. Sturny, L. Crivelli, S. Edwards-Garavoglia, A. Spranger, F. Wittenbecher, and W. Quentin, "Switzerland: Health System Review", Health systems in transition, 17(4), 2015, 1-288, xix.

[28] Reychav, I., R. Kumi, R. Sabherwal, and J. Azuri, "Using tablets in medical consultations: Single loop and double loop learning processes", Computers in Human Behavior, 61, 2016, pp. 415-426.

[29] Rieger, K.L., T.F. Hack, K. Beaver, and P. Schofield, "Should consultation recording use be a practice standard? A systematic review of the effectiveness and implementation of consultation recordings", Psychooncology, 27(4), 2018, pp. 1121-1128.

[30] Rosson, M.B. and J.M. Carroll, Usability engineering: Scenario-based development of human-computer interaction, 1st edn., Academic Press, San Fancisco, 2010.

[31] Schmidt-Rauch, S. and G. Schwabe, "Designing for mobile value co-creation - the case of travel counselling", Electronic Markets, 24(1), 2014, pp. 5-17.

[32] Schultze, U. and M. Avital, "Designing interviews to generate rich data for information systems research", Information and Organization, 21(1), 2011, pp. 1-16.

[33] Svenstrup, D., H.L. Jørgensen, and O. Winther, "Rare disease diagnosis: A review of web search, social media and large-scale data-mining approaches", Rare diseases (Austin, Tex.), 3(1), 2015, e1083145.

[34] van den Brink-Muinen, A., A.M. van Dulmen, J.M. Bensing, H.-I. Maaros, H. Tähepöld, L. Plawecka, Z.J. Krol, S.C. Oana, M. Boros, U. Satterlund-Larsson, and B.M. Bengtsson, Eurocommunication II: A comparative study between countries in Central- and Western-Europe on doctor-patient communication in general practice, NIVEL, 2003.

[35] van den Brink-Muinen, A., P.F.M. Verhaak, J.M. Bensing, O. Bahrs, M. Deveugele, L. Gask, N. Mead, F. Leiva-Fernandez, A. Perez, and V. Messerli, The Eurocommunication Study: An international comparative study in six European countries on doctor-patien communication, NIVEL, 1999.

[36] Vermeire, E., H. Hearnshaw, P. van Royen, and J. Denekens, "Patient adherence to treatment: Three decades of research. A comprehensive review", Journal of Clinical Pharmacy and Therapeutics, 26(5), 2001, pp. 331-342.

[37] Zolnierek, K.B.H. and M.R. Dimatteo, "Physician communication and patient adherence to treatment: A meta-analysis", Medical care, 47(8), 2009, pp. 826-834. 